\title{
Multiple Criteria Decision Analysis Using Correlation-Based Precedence Indices Within Pythagorean Fuzzy Uncertain Environments
}

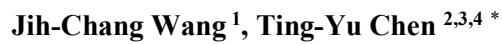 \\ ${ }^{1}$ Department of Information Management, Chang Gung University, \\ No. 259, Wenhua 1st Rd., Guishan District \\ Taoyuan City 33302, Taiwan \\ E-mail:qpo@mail.cgu.edu.tw \\ ${ }^{2}$ Graduate Institute of Business and Management, Chang Gung University \\ ${ }^{3}$ Department of Industrial and Business Management, Chang Gung University \\ ${ }^{4}$ Department of Nursing, Linkou Chang Gung Memorial Hospital \\ No. 259, Wenhua 1st Rd., Guishan District \\ Taoyuan City 33302, Taiwan \\ E-mail: tychen@mail.cgu.edu.tw
}

Received December 20, 2017

Accepted March 24, 2018

\begin{abstract}
The theory of Pythagorean fuzzy sets possesses significant advantages in handling vagueness and complex uncertainty. Additionally, Pythagorean fuzzy information is useful to simulate the ambiguous nature of subjective judgments and measure the fuzziness and imprecision more flexibly. The aim of this research is to develop an effective assignment-based method using a novel concept of correlation-based precedence indices for conducting multiple criteria decision analysis within the Pythagorean fuzzy uncertain environment. Based on the ideas of information energy and correlations, this paper defines a novel concept of correlation-based precedence indices in the Pythagorean fuzzy context and discusses their desirable properties. Next, this paper presents some useful concepts of discordance indicators, weighted discordance indicators, comprehensive discordance indicators, and comprehensive discordance indices to construct a novel assignment model for acquiring a comprehensive ranking of candidate alternatives. As an application of the proposed assignment-based method, a practical example concerning a financing decision of working capital policies is provided to demonstrate its practicality and effectiveness.
\end{abstract}

Keywords: Pythagorean fuzzy set, correlation-based precedence index, multiple criteria decision analysis, discordance indicator, comprehensive discordance index, assignment model.

\section{Introduction}

Decision-making information provided by decision makers is often imprecise and uncertain because of a lack of data, time pressure, or the decision maker's limited attention and information-processing capabilities. Accordingly, research pertaining to multiple criteria decision analysis (MCDA) problems has often been performed within a fuzzy uncertain environment ${ }^{1,4,11,18}$

The concept of Pythagorean fuzzy (PF) sets, which was introduced by Yager $^{23}$ and Yager and Abbasoveve, is a useful and valuable extension of Atanassov's intuitionistic fuzzy sets ${ }^{2}$, in which the sum of the degree of membership and the degree of non-membership is less than or equal to one. Intuitionistic fuzzy sets are the most widely used type of nonstandard fuzzy models because of their great ability to handle imprecise and ambiguous information ${ }^{3,4,18}$. In a similar manner of the intuitionistic fuzzy model, PF sets are also characterized by degrees of membership and non-membership. However, their sum is not required to be less than one. Instead, the square sum of degrees of membership and non-membership is less than or equal to one $e^{8,12,17,25}$. Because of the relaxed constraint conditions, PF sets are

\footnotetext{
*E-mail: tychen@mail.cgu.edu.tw.
}

Copyright (C) 2018, the Authors. Published by Atlantis Press.

This is an open access article under the CC BY-NC license (http://creativecommons.org/licenses/by-nc/4.0/). 
capable of managing more-complex uncertainty in realworld decision situations than intuitionistic fuzzy sets $^{6,7,25}$. From this perspective, PF sets are becoming increasingly popular in the MCDA field by the day ${ }^{5-7,15}$.

Because PF sets possess superior ability to reflect the ambiguous nature of subjective judgments and model highly uncertain information in realistic applications, numerous valuable methods have been developed to solve MCDA problems within the PF environment. For example, Garg ${ }^{10}$ presented some series of geometric-aggregated operators within the PF environment and employed Einstein t-norm and $t$ conorm for MCDA. By combining PF sets with hesitant fuzzy sets, Liang and $\mathrm{Xu}^{12}$ extended the technique for order preference by similarity to ideal solution (TOPSIS) to the hesitant PF environment. Mohagheghi et al. ${ }^{13}$ introduced a last aggregation evaluating approach based on a group decision-making procedure and PF sets to enhance decision flexibility. Rahman et al. ${ }^{16}$ developed useful PF weighted geometric aggregation operators to solve a group decision-making problem concerning plant location selection. Wei ${ }^{19}$ proposed some PF interaction aggregation operators to solve MCDA problems in the $\mathrm{PF}$ context. $\mathrm{Xu}$ et al. ${ }^{21}$ proposed $\mathrm{PF}$ induced generalized ordered weighted averaging (OWA) operators to manage group decision-making problems.

Based on useful concepts of displaced and fixed remoteness indices, $\mathrm{Chen}^{7}$ developed a novel VlseKriterijumska Optimizacija I Kompromisno Resenje (i.e., multicriteria optimization and compromise solution) (VIKOR) method for MCDA involving Pythagorean fuzzy information. Wei and $\mathrm{Lu}^{20}$ utilized power aggregation operators to develop some useful PF power aggregation operators for addressing MCDA problems. Xue et al. ${ }^{22}$ proposed the linear programming technique for multidimensional analysis of preference (LINMAP) based on the entropy theory and PF sets, and they applied the PF LINMAP to solve multiple attribute group decision-making problems.

Considering the powerfulness of the PF theory in tackling imprecise and ambiguous information, the purpose of this paper is to propose an assignment-based MCDA method using correlation-based precedence indices for addressing MCDA problems under complex uncertainty based on PF sets. Concretely speaking, this paper first presents a valuable concept of correlationbased precedence indices that can fully take into account the amount of information associated with PF sets and can effectively discriminate among evaluative ratings under complex uncertainty. In the PF context, this paper subsequently discusses some valuable properties of the developed correlation-based precedence index in detail. By use of correlation-based precedence indices, this paper constructs some useful concepts of a discordance indicator, a weighted discordance indicator, a comprehensive discordance indicator, and a comprehensive discordance index within the PF environment. Based on these valuable concepts and a permutation matrix, this paper establishes an assignment model for the purpose of addressing MCDA problems involving PF information. Furthermore, an effective algorithm is provided to help decision makers manage vagueness and uncertainty and conduct multiple criteria evaluation and selection in the PF context. Finally, a practical example is provided to illustrate the application of the proposed methodology and demonstrate its practicality and effectiveness.

The remainder of this paper is organized as follows. Section 2 briefly introduces some basic concepts related to PF sets that are used throughout this article. Section 3 develops a new concept of correlation-based precedence indices and investigates some interesting properties. Section 4 formulates an MCDA problem under complex uncertainty based on PF information. Section 5 proposes a novel assignment-based MCDA method for acquiring a comprehensive ranking of competing alternatives. Section 6 applies the developed methodology to a practical problem regarding a financing problem of working capital policies in order to demonstrate its feasibility and applicability. Section 7 investigates a comparison of the effects between the Pythagorean fuzzy and intuitionistic fuzzy approaches through a sensitivity analysis in the context of intuitionistic fuzziness. Finally, Section 8 presents the conclusions.

\section{Basic Concept of PF Sets}

This section first briefly reviews some basic concepts related to PF sets that are used throughout this article.

Definition 1. ${ }^{14,23,24}$ A PF set $P$ is defined as a set of ordered pairs of membership and non-membership in a finite universe of discourse $X$ and is given as follows:

$$
P=\left\{\left\langle x, \mu_{P}(x), v_{P}(x)\right\rangle \mid x \in X\right\},
$$


which is characterized by the degree of membership $\mu_{p}$ : $X \rightarrow[0,1]$ and the degree of non-membership $v_{P}: X \rightarrow[0$, 1] of the element $x \in X$ to the set $P$ with the condition:

$$
0 \leq\left(\mu_{P}(x)\right)^{2}+\left(v_{P}(x)\right)^{2} \leq 1 .
$$

Let $p=\left(\mu_{P}(x), v_{P}(x)\right)$ denote a PF value. The degree of indeterminacy relative to $P$ for each $x \in X$ is defined as follows:

$$
\pi_{P}(x)=\sqrt{1-\left(\mu_{P}(x)\right)^{2}-\left(v_{P}(x)\right)^{2}} .
$$

It is worth noting that the PF value $\left(\mu_{P}(x), v_{P}(x)\right)$ is reduced to an intuitionistic fuzzy value if $\mu_{P}(x)+v_{P}(x)$ $\leq 1$. Fig. 1 provides a convenient geometrical interpretation regarding the spaces of $\mathrm{PF}$ and intuitionistic fuzzy values. It is obvious to see that the main difference between a PF value and an intuitionistic fuzzy value is their different constraint conditions. As shown in Fig.1, the space of PF values is apparently larger than that of intuitionistic fuzzy values. As a result, PF sets can not only depict uncertain information, which intuitionistic fuzzy sets can capture, but also model more imprecise and ambiguous information, which the latter cannot describe. Because of the relaxed constraint condition $\left(\mu_{P}(x)\right)^{2}+\left(v_{P}(x)\right)^{2} \leq 1, \mathrm{PF}$ sets are more precise in the modeling of vagueness and complex uncertainty in MCDA problems compared with intuitionistic fuzzy sets.

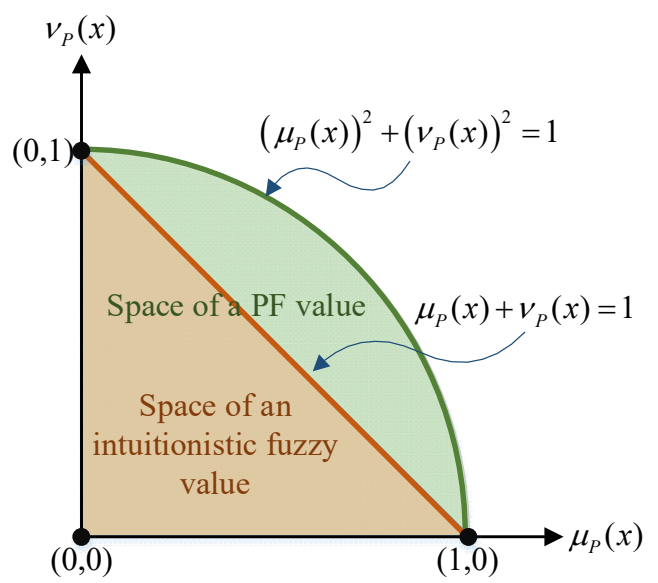

Fig. 1. Comparison of spaces for a PF value and an intuitionistic fuzzy value.
Definition 2. ${ }^{7,9,13,14}$ Let $p_{1}, p_{2}$, and $p$ be three PF values in $X$ and $\alpha \geq 0$. Selected basic operations are defined as follows:

$$
\begin{gathered}
p_{1} \vee p_{2}=\left(\max \left\{\mu_{P_{1}}(x), \mu_{P_{2}}(x)\right\},\right. \\
\left.\min \left\{v_{P_{1}}(x), v_{P_{2}}(x)\right\}\right), \\
p_{1} \wedge p_{2}=\left(\min \left\{\mu_{P_{1}}(x), \mu_{P_{2}}(x)\right\},\right. \\
\left.\max \left\{v_{P_{1}}(x), v_{P_{2}}(x)\right\}\right), \\
p_{1} \oplus p_{2}=\left(\left(\left(\mu_{P_{1}}(x)\right)^{2}+\left(\mu_{P_{2}}(x)\right)^{2}-\left(\mu_{P_{1}}(x)\right)^{2}\right.\right. \\
\left.\left.\cdot\left(\mu_{P_{2}}(x)\right)^{2}\right)^{0.5}, v_{P_{1}}(x) \cdot v_{P_{2}}(x)\right), \\
p_{1}^{\otimes} p_{2}=\left(\mu_{P_{1}}(x) \cdot \mu_{P_{2}}(x),\left(\left(v_{P_{1}}(x)\right)^{2}+\left(v_{P_{2}}(x)\right)^{2}\right.\right. \\
\left.\left.-\left(v_{P_{1}}(x)\right)^{2} \cdot\left(v_{P_{2}}(x)\right)^{2}\right)^{0.5}\right), \\
\alpha \cdot p=\left(\sqrt{1-\left(1-\left(\mu_{P}(x)\right)^{2}\right)^{\alpha}},\left(v_{P}(x)\right)^{\alpha}\right), \\
p^{\alpha=}\left(\left(\mu_{P}(x)\right)^{\alpha}, \sqrt{1-\left(1-\left(v_{P}(x)\right)^{2}\right)^{\alpha}}\right) .
\end{gathered}
$$

\section{Correlation-Based Precedence Index}

Inspired by the idea of correlation coefficients for PF sets $^{9}$, this section introduces a novel concept of correlation-based precedence indices within the PF environment and investigates their useful and desirable properties.

It is worthwhile to mention that the core concept of the proposed methodology is an extended definition of correlation coefficients in the PF context. The concept of correlation coefficients is one of the most used indices in statistics and engineering sciences. With the aid of a measure of interdependency, correlation can reflect the joint relationship between two variables and indicate how well two variables move together in a linear fashion. However, the existing correlation coefficients based on the probability theory cannot be employed within the PF environment. In particular, massive fuzzy uncertainty exists in a complicated decision-making system, which leads to the difficulty in exact estimations of the probability of events. Accordingly, the correlation coefficients yielded by the 
probabilistic approach have limitations to deal with built-in uncertainties contained in the PF information.

Considering the usefulness of correlation coefficients in many real-world decision-making problems, this paper proposes a novel correlation coefficient to measure the relationship between two PF values. Different from the classical definition, pairs of membership, non-membership, and indeterminacy degrees within the PF values will be considered during formulation of PF correlation coefficients. Based on the extended correlation coefficient, a useful concept of correlation-based precedence indices is then constructed in this section. These new concepts can facilitate to establish an easy-to-use assignment-based model to manage MCDA problems with PF information. As can be expected, the results based on the proposed correlation coefficients and correlation-based precedence indices can provide useful and influential information to decision makers and hence the corresponding MCDA approach is adequate to account for highly complicated uncertainties within the PF environment.

Definition 3. Let $p$ be a PF value in $X$. The information energy $T$ of $p$ is defined as follows:

$$
T(p)=\left(\mu_{P}(x)\right)^{4}+\left(v_{P}(x)\right)^{4}+\left(\pi_{P}(x)\right)^{4} .
$$

Theorem 1. The information energy $T$ of a PF value $p$ satisfies the following properties:

(T1.1) $0<T(p) \leq 1$;

(T1.2) $T((1,0))=T((0,1))=T((0,0))=1$.

Proof. (T1.1) can be directly inferred according to Definition 1. (T1.2) is evident from Definition 3.

Definition 4. Let $p_{1}$ and $p_{2}$ be two PF values in $X$. The correlation $R$ between $p_{1}$ and $p_{2}$ is defined as follows:

$$
\begin{aligned}
R\left(p_{1}, p_{2}\right)= & \left(\mu_{P_{1}}(x)\right)^{2} \cdot\left(\mu_{P_{2}}(x)\right)^{2}+\left(v_{P_{1}}(x)\right)^{2} \\
& \cdot\left(v_{P_{2}}(x)\right)^{2}+\left(\pi_{P_{1}}(x)\right)^{2} \cdot\left(\pi_{P_{2}}(x)\right)^{2} .
\end{aligned}
$$

Theorem 2. The correlation $R$ between two PF values satisfies the following properties:

(T2.1) $R(p, p)=T(p)$;

(T2.2) $0 \leq R\left(p_{1}, p_{2}\right) \leq 1$;

(T2.3) $R\left(p_{1}, p_{2}\right)=R\left(p_{2}, p_{1}\right)$.
Proof. (T2.1)-(T2.3) are straightforward.

Definition 4. Let $p_{1}$ and $p_{2}$ be two PF values in $X$. The correlation coefficient $K$ between $p_{1}$ and $p_{2}$ is defined as follows:

$$
K\left(p_{1}, p_{2}\right)=\frac{R\left(p_{1}, p_{2}\right)}{\sqrt{T\left(p_{1}\right) \cdot T\left(p_{2}\right)}} .
$$

Theorem 3. The correlation coefficient $K$ between two $P F$ values $p_{1}$ and $p_{2}$ satisfies the following properties:

(T3.1) $K\left(p_{1}, p_{2}\right)=K\left(p_{2}, p_{1}\right)$;

(T3.2) $0 \leq K\left(p_{1}, p_{2}\right) \leq 1$;

(T3.3) $K\left(p_{1}, p_{2}\right)=1$ if $p_{1}=p_{2}$.

Proof. (T3.1) is obvious.

(T3.2) According to (T1.1) and (T2.2), it can be easily obtained that $K\left(p_{1}, p_{2}\right) \geq 0$. From Definition 4 , one has:

$$
\begin{aligned}
\left(R\left(p_{1}, p_{2}\right)\right)^{2}= & \left(\left(\mu_{P_{1}}(x)\right)^{2} \cdot\left(\mu_{P_{2}}(x)\right)^{2}+\left(v_{P_{1}}(x)\right)^{2}\right. \\
& \left.\cdot\left(v_{P_{2}}(x)\right)^{2}+\left(\pi_{P_{1}}(x)\right)^{2} \cdot\left(\pi_{P_{2}}(x)\right)^{2}\right)^{2} .
\end{aligned}
$$

Applying the Cauchy-Schwarz inequality, one obtains:

$$
\begin{aligned}
& \left(\left(\mu_{P_{1}}(x)\right)^{2} \cdot\left(\mu_{P_{2}}(x)\right)^{2}+\left(v_{P_{1}}(x)\right)^{2} \cdot\left(v_{P_{2}}(x)\right)^{2}\right. \\
& \left.+\left(\pi_{P_{1}}(x)\right)^{2} \cdot\left(\pi_{P_{2}}(x)\right)^{2}\right)^{2} \\
& \leq\left[\left(\left(\mu_{P_{1}}(x)\right)^{2}\right)^{2}+\left(\left(v_{P_{1}}(x)\right)^{2}\right)^{2}+\left(\left(\pi_{P_{1}}(x)\right)^{2}\right)^{2}\right] \\
& \quad \cdot\left[\left(\left(\mu_{P_{2}}(x)\right)^{2}\right)^{2}+\left(\left(v_{P_{2}}(x)\right)^{2}\right)^{2}+\left(\left(\pi_{P_{2}}(x)\right)^{2}\right)^{2}\right] \\
& =\left(\left(\mu_{P_{1}}(x)\right)^{4}+\left(v_{P_{1}}(x)\right)^{4}+\left(\pi_{P_{1}}(x)\right)^{4}\right) \\
& \cdot\left(\left(\mu_{P_{2}}(x)\right)^{4}+\left(v_{P_{2}}(x)\right)^{4}+\left(\pi_{P_{2}}(x)\right)^{4}\right) .
\end{aligned}
$$

Thus, it is clear that:

$$
\left(R\left(p_{1}, p_{2}\right)\right)^{2} \leq T\left(p_{1}\right) \cdot T\left(p_{2}\right),
$$

which yields $K\left(p_{1}, p_{2}\right) \leq 1$. Therefore, (T3.2) is valid. (T3.3) It is known that $\mu_{P_{1}}(x)=\mu_{P_{2}}(x)$ and $v_{P_{1}}(x)$ $=v_{P_{2}}(x)$ because $p_{1}=p_{2}$. By use of (T2.1), one has $R\left(p_{1}, p_{1}\right)=T\left(p_{1}\right)$. Moreover, $T\left(p_{1}\right)=T\left(p_{2}\right)$ Therefore,

$$
K\left(p_{1}, p_{2}\right)=\frac{T\left(p_{1}\right)}{\sqrt{T\left(p_{1}\right) \cdot T\left(p_{1}\right)}}=1 .
$$


This completes the proof.

Definition 5. Let $p$ be a PF value in $X$, and assume that $p \neq(0,0)$ without loss of generality. The correlationbased precedence index $I$ of $p$ is defined as follows:

$$
I(p)=\frac{K(p,(1,0))}{K(p,(1,0))+K(p,(0,1))},
$$

where $(1,0)$ and $(0,1)$ are the largest and smallest PF values, respectively.

Theorem 4. The correlation-based precedence index I(p) can be determined as follows:

$$
I(p)=\frac{\left(\mu_{P}(x)\right)^{2}}{1-\left(\pi_{P}(x)\right)^{2}} .
$$

Proof. According to (T1.2), it can be obtained that:

$$
\begin{aligned}
I(p)= & \frac{\frac{R(p,(1,0))}{\sqrt{T(p) \cdot T((1,0))}}}{\sqrt{T(p,(1,0))}+\frac{R(p,(0,1))}{\sqrt{T(p) \cdot T((0,1))}}} \\
= & \frac{\frac{R(p,(1,0))}{\sqrt{T(p)}}}{\frac{R(p,(1,0))}{\sqrt{T(p)}}+\frac{R(p,(0,1))}{\sqrt{T(p)}}} \\
= & \frac{R(p,(1,0))}{R(p,(1,0))+R(p,(0,1))} .
\end{aligned}
$$

Because $\quad R(p,(1,0))=\left(\mu_{P}(x)\right)^{2} \quad$ and $\quad R(p,(0,1))=$ $\left(v_{P}(x)\right)^{2}$ from Definition 4 , one has:

$$
I(p)=\frac{\left(\mu_{P}(x)\right)^{2}}{\left(\mu_{P}(x)\right)^{2}+\left(v_{P}(x)\right)^{2}}=\frac{\left(\mu_{P}(x)\right)^{2}}{1-\left(\pi_{P}(x)\right)^{2}} .
$$

This establishes the theorem.

Theorem 5. The correlation-based precedence index I of a PF value $p$ satisfies the following properties:

(T5.1) $0 \leq I(p) \leq 1$;

(T5.2) $I(p)=0$ if and only if $\mu_{P}(x)=0$;

(T5.3) $I(p)=1$ if and only if $v_{P}(x)=0$;

(T5.4) $I(p)=0$ if $p=(0,1)$;

(T5.5) $I(p)=1$ if $p=(1,0)$.

Proof. (T5.1) is straightforward because $K(p,(1,0)) \geq 0$, $K(p,(0,1)) \geq 0$, and $K(p,(1,0)) \leq K(p,(1,0))+K(p,(0,1))$.
(T5.2) For the necessity, if $I(p)=0$, then it follows that the condition of $\mu_{P}(x)=0$ must be fulfilled according to Theorem 4. For the sufficiency, if $\mu_{P}(x)=0$, then it is easy to obtain that $I(p)=0$. Hence, (T5.2) is correct.

(T5.3) For the necessity, if $I(p)=1$, then it implies that $\left(\mu_{P}(x)\right)^{2}=1-\left(\pi_{P}(x)\right)^{2}=\left(\mu_{P}(x)\right)^{2}+\left(v_{P}(x)\right)^{2}$ by use of Theorem 4. This indicates that $v_{P}(x)=0$. For the sufficiency, if $v_{P}(x)=0$, then $1-\left(\pi_{P}(x)\right)^{2}=\left(\mu_{P}(x)\right)^{2}$, which yields that $I(p)=1$. Thus, (T5.3) is correct. (T5.4) and (T5.5) can be easily inferred from (T5.2) and (T5.3), respectively, which completes the proof.

\section{MCDA Problem Under PF Uncertainty}

The discussed problems in this paper mainly are a type of decision-making problems which have limited numbers of candidate alternatives evaluated on multiple criteria. Additionally, in view that there are many realworld situations where due to insufficiency in information availability, PF sets are appropriate to deal with such problems under complex uncertainty.

Consider an MCDA problem in which $Z=\left\{z_{1}, z_{2}\right.$, $\left.\cdots, z_{m}\right\}$ is a set of $m(m \geq 2)$ candidate alternatives and $C=\left\{c_{1}, c_{2}, \cdots, c_{n}\right\}$ is a finite set of $n(n \geq 2)$ criteria. For numerous practical MCDA problems, the decision maker's evaluations related to grades of importance of criteria and evaluative ratings of alternatives with respect to each criterion are often expressed by linguistic terms comprising vagueness and uncertainty. These uncertain, vague and hesitant judgments provided by the decision maker can be represented more comprehensively by using the PF sets. For example, $\mathrm{Chen}^{7}$ suggested a PF linguistic rating system to transform five- and seven-point linguistic rating scales into suitable PF values.

By use of a linguistic rating system, the evaluative rating of an alternative $z_{i} \in Z$ with respect to a criterion $c_{j} \in C$ can be expressed as a PF value $p_{i j}=\left(\mu_{i j}, v_{i j}\right)$, such that $\mu_{i j} \in[0,1], v_{i j} \in[0,1]$, and $0 \leq\left(\mu_{i j}\right)^{2}+\left(v_{i j}\right)^{2}$ $\leq 1$. In particular, $\mu_{i j}$ and $v_{i j}$ represent the degrees to which $z_{i}$ performs well and poorly, respectively, in terms of $c_{j}$. The indeterminacy degree that corresponds to each $p_{i j}$ is computed as $\pi_{i j}=\sqrt{1-\left(\mu_{i j}\right)^{2}-\left(v_{i j}\right)^{2}}$. The MCDA problem within the PF environment can be concisely expressed in the following PF decision matrix: 


$$
\begin{aligned}
& \mathrm{p}=\left[p_{i j}\right]_{m \times n} \\
& \begin{array}{cccc}
c_{1} & c_{2} & \cdots & c_{n}
\end{array}
\end{aligned}
$$

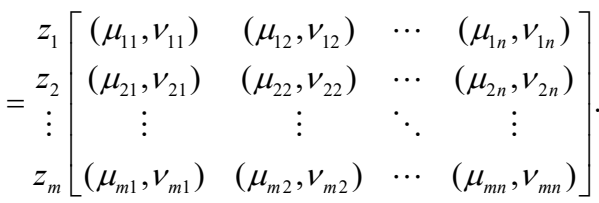

In the PF context, let a PF value $w_{j}=\left(\omega_{j}, \varpi_{j}\right)$ denote the importance weight of criterion $c_{j} \in C$, such that $\omega_{j} \in[0,1], \varpi_{j} \in[0,1]$, and $0 \leq\left(\omega_{j}\right)^{2}+\left(\varpi_{j}\right)^{2} \leq 1$. The indeterminacy degree $\tau_{j}$ that corresponds to each $w_{j}$ is determined as $\sqrt{1-\left(\omega_{j}\right)^{2}-\left(\varpi_{j}\right)^{2}}$.

Having formulating the MCDA problem involving $\mathrm{PF}$ information, the next part of this research is to postulate and define a new assignment model by utilizing the developed concept of correlation-based precedence indices.

\section{A Novel Assignment-Based MCDA Method}

Based on the correlation-based precedence index, this section attempts to establish a novel assignment-based MCDA method to determine a comprehensive ranking of candidate alternatives that is in the closest agreement with the criterion-wise precedence ranks among alternatives.

As demonstrated in the previous theorems, the correlation-based precedence index within the PF environment possesses certain useful and interesting properties. Consider a $\mathrm{PF}$ evaluative rating $p_{i j}$ in the $\mathrm{PF}$ decision matrix p. The larger the $I\left(p_{i j}\right)$ value is, the better the performance on the evaluation result it is, and the greater the preference is for $p_{i j}$. In contrast, the smaller the $I\left(p_{i j}\right)$ value is, the worse the performance on the evaluation result it is, and the lesser the preference is for $p_{i j}$. Additionally, the same $I\left(p_{i j}\right)$ value represents indifferent preference. Consequently, the criterion-wise precedence ranks among competing alternatives can be precisely determined in descending order of the $I\left(p_{i j}\right)$ values.

Definition 6. Let $p_{i_{1} j}$ and $p_{i_{2} j}$ be the PF evaluative ratings of two alternatives $z_{i_{1}}$ and $z_{i_{2}}$, respectively, regarding a criterion $c_{j}$ in $\mathrm{p}$. The following precedence relationships between $z_{i_{1}}$ and $z_{i_{2}}$ are defined according to their correlation-based precedence indices:
(D6.1) $z_{i_{1}}$ is prior to $z_{i_{2}}$ with respect to $c_{j}$ if $I\left(p_{i_{1} j}\right)>$ $I\left(p_{i_{2} j}\right)$;

(D6.2) $z_{i_{1}}$ is indifferent to $z_{i_{2}}$ with respect to $c_{j}$ if $I\left(p_{i_{1} j}\right)=I\left(p_{i_{2} j}\right)$;

(D6.3) $z_{i_{1}}$ is posterior to $z_{i_{2}}$ with respect to $c_{j}$ if $I\left(p_{i_{1} j}\right)<I\left(p_{i_{2} j}\right)$.

Based on the precedence relationships obtained from Definition 6, the precedence ranks in terms of each criterion can be acquired with the aid of correlationbased precedence indices. Let $r_{i j}$ denote the precedence rank of an alternative $z_{i} \in Z \quad(i \in\{1,2, \cdots, m\})$ with respect to criterion $c_{j} \in C(j \in\{1,2, \cdots, n\})$. In case of the occurrence of ties among the criterion-wise precedence ranks, a mean rank should be assigned to the tied alternatives. For example, when two alternatives are tied for the fourth rank, a precedence rank of 4.5 $(=(4+5) / 2)$ should be assigned. In this way, the rank evaluation matrix $r$ can be established as follows:

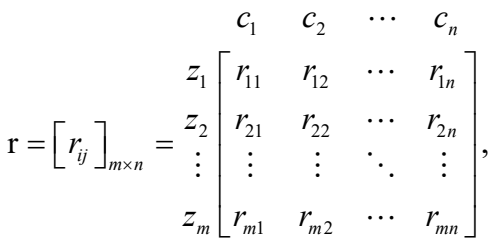

where $1 \leq r_{i j} \leq m$ and $\sum_{i=1}^{m} r_{i j}=m(m+1) / 2$ for all $j \in\{1,2, \cdots, n\}$.

By using the proposed concept of correlation-based precedence indices and the criterion-wise precedence ranks, this paper attempts to develop some useful indicators to determine a comprehensive ranking for all competing alternatives. In particular, the comprehensive ranking is that ranking which is in best agreement with the obtained rankings based on criterion-wise precedence relationships and the criterion-wise performance based on correlation-based precedence indices. Such a comprehensive ranking can form an objective basis for arriving at a compromise of all precedence ranks in the rank evaluation matrix $r$.

Definition 7. Given the set of alternatives $Z$ and the set of criteria $C$, let $r_{i j}$ be the criterion-wise precedence rank of $z_{i} \in Z$ according to $c_{j} \in C$. The discordance indicator $\hat{d}_{i}^{0 k}$ for $z_{i}$ to become the $k$-th comprehensive rank is defined as the sum of absolute distances between $r_{i j}$ and $k$ : 


$$
\widehat{d}_{i}^{0 k}=\sum_{j=1}^{n}\left|r_{i j}-k\right|
$$

Moreover, by coupling the PF importance weight $w_{j}$ into $\bar{d}_{i}^{0 k}$, the weighted discordance indicator $\hat{d}_{i}^{k}$ is defined as the weighted sum of absolute distances for $z_{i}$ to be ranked $k$-th:

$$
\widehat{d}_{i}^{k}=\bigoplus_{j=1}^{n}\left|r_{i j}-k\right| \cdot w_{j} .
$$

Furthermore, based on the criterion-wise performance, the comprehensive discordance indicator $d_{i}^{k}$ that incorporates the correlation-based precedence index $I\left(p_{i j}\right)$ into $\hat{d}_{i}^{k}$ is defined as the comprehensive sum of absolute distances for $z_{i}$ to be ranked $k$-th:

$$
d_{i}^{k}=\oplus_{j=1}^{n}\left(I\left(p_{i j}\right)\left|r_{i j}-k\right|\right) \cdot w_{j} .
$$

Theorem 6. The comprehensive discordance indicator $d_{i}^{k}$ is a PF value that can be computed in the following manner:

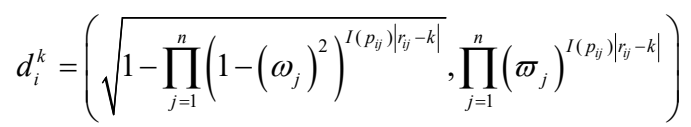

for $i, k=1,2, \cdots, m$. Denote $d_{i}^{k}=\left(\delta_{i}^{k}, \sigma_{i}^{k}\right)$ for brevity.

Proof. By mathematical induction on $n$, the following results are obtained. First, Eq. (20) holds for $n=2$ because:

$$
\begin{aligned}
& d_{i}^{k}=\left(\left(I\left(p_{i 1}\right)\left|r_{i 1}-k\right|\right) \cdot w_{1}\right) \oplus\left(\left(I\left(p_{i 2}\right)\left|r_{i 2}-k\right|\right) \cdot w_{2}\right) \\
& =\left(\sqrt{1-\left(1-\left(\omega_{1}\right)^{2}\right)^{I\left(p_{i 1}\right)\left|r_{i 1}-k\right|}},\left(\varpi_{1}\right)^{I\left(p_{i 1}\right)\left|r_{i 1}-k\right|}\right) \\
& \oplus\left(\sqrt{1-\left(1-\left(\omega_{2}\right)^{2}\right)^{I\left(p_{i 2}\right)\left|r_{i 2}-k\right|}},\left(\varpi_{2}\right)^{I\left(p_{i 2}\right)\left|r_{i 2}-k\right|}\right) \\
& =\left(\left(1-\left(1-\left(\omega_{1}\right)^{2}\right)^{I\left(p_{i 1}\right)\left|r_{i 1}-k\right|}+1-\left(1-\left(\omega_{2}\right)^{2}\right)^{I\left(p_{i 2}\right)\left|r_{i 2}-k\right|}\right.\right. \\
& -\left(1-\left(1-\left(\omega_{1}\right)^{2}\right)^{I\left(p_{i 1}\right)\left|r_{i 1}-k\right|}\right) \\
& \left.\cdot\left(1-\left(1-\left(\omega_{2}\right)^{2}\right)^{I\left(p_{i 2}\right)\left|r_{i 2}-k\right|}\right)\right)^{0.5} \text {, } \\
& \left.\left(\varpi_{1}\right)^{I\left(p_{i 1}\right)\left|r_{i 1}-k\right|} \cdot\left(\varpi_{2}\right)^{I\left(p_{i 2}\right)\left|r_{i 2}-k\right|}\right)
\end{aligned}
$$

$$
\begin{aligned}
= & \left(\left(1-\left(1-\left(1-\left(1-\left(\omega_{1}\right)^{2}\right)^{I\left(p_{i 1}\right)\left|r_{i 1}-k\right|}\right)\right)\right.\right. \\
& \left.\cdot\left(1-\left(1-\left(1-\left(\omega_{2}\right)^{2}\right)^{I\left(p_{i 2}\right)\left|r_{i 2}-k\right|}\right)\right)\right)^{0.5}, \\
& \left.\left(\varpi_{1}\right)^{I\left(p_{i 1}\right)\left|r_{i 1}-k\right|} \cdot\left(\varpi_{2}\right)^{I\left(p_{i 2}\right)\left|r_{i 2}-k\right|}\right) \\
= & \left(\sqrt{\left.1-\prod_{j=1}^{2}\left(1-\left(\omega_{j}\right)^{2}\right)^{I\left(p_{i j}\right) r_{i j}-k \mid}, \prod_{j=1}^{2}\left(\varpi_{j}\right)^{I\left(p_{i j}\right)\left|r_{i j}-k\right|}\right) .} .\right.
\end{aligned}
$$

Assume that Eq. (20) holds for $n=\varphi$, i.e.:

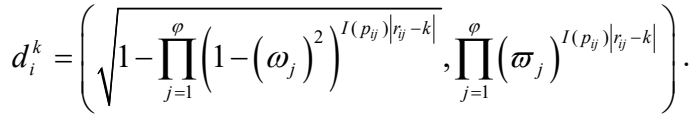

When $n=\varphi+1$, one obtains:

$$
\begin{aligned}
& d_{i}^{k}=\left(\bigoplus_{j=1}^{\varphi}\left(I\left(p_{i j}\right)\left|r_{i j}-k\right|\right) \cdot w_{j}\right) \\
& \oplus\left(\left(I\left(p_{i, \varphi+1}\right)\left|r_{i, \varphi+1}-k\right|\right) \cdot w_{\varphi+1}\right)
\end{aligned}
$$

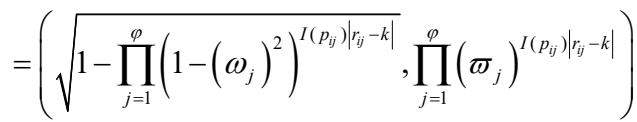

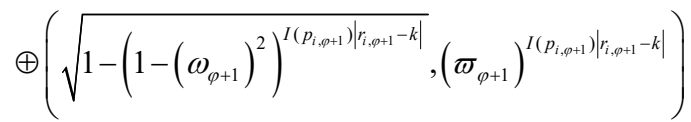

$$
\begin{aligned}
& =\left(1-\prod_{j=1}^{\varphi}\left(1-\left(\omega_{j}\right)^{2}\right)^{I\left(p_{i j}\right) r_{i j}-k \mid}+1\right. \\
& -\left(1-\left(1-\left(1-\left(\omega_{\varphi+1}\right)^{2}\right)^{I\left(p_{i, \varphi+1}\right)\left|r_{i, \varphi+1}-k\right|}\right)\right) \\
& -\left(1-\prod_{j=1}^{\varphi}\left(1-\left(\omega_{j}\right)^{2}\right)^{I\left(p_{i j}\right)\left|r_{i j}-k\right|}\right) \\
& \left.\cdot\left(1-\left(1-\left(1-\left(1-\left(\omega_{\varphi+1}\right)^{2}\right)^{\left.I\left(p_{i, \varphi+1}\right)\right|_{i, \varphi+1}-k \mid}\right)\right)\right)\right)^{0.5}, \\
& \left.\left(\prod_{j=1}^{\varphi}\left(\varpi_{j}\right)^{\left.I\left(p_{i j}\right)\right|_{i j}-k \mid}\right) \cdot\left(\varpi_{\varphi+1}\right)^{I\left(p_{i, \varphi+1}\right)\left|r_{i, \varphi+1}-k\right|}\right)
\end{aligned}
$$

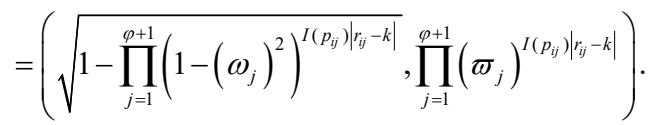

Thus, Eq. (20) holds for $n=\varphi+1$. Therefore, Eq. (20) holds for all $n$. Because $0 \leq\left(\omega_{j}\right)^{2}+\left(\varpi_{j}\right)^{2} \leq 1$ for all $j$, it is clear that: 


$$
\begin{aligned}
& \left(\delta_{i}^{k}\right)^{2}+\left(\sigma_{i}^{k}\right)^{2} \\
& =\left(\sqrt{1-\prod_{j=1}^{n}\left(1-\left(\omega_{j}\right)^{2}\right)^{I\left(p_{i j}\right) r_{i j}-k \mid}}\right)^{2}+\left(\prod_{j=1}^{n}\left(\varpi_{j}\right)^{I\left(p_{i j}\right) r_{r_{j}}-k \mid}\right)^{2} \\
& =1-\prod_{j=1}^{n}\left(1-\left(\omega_{j}\right)^{2}\right)^{I\left(p_{i j}\right) r_{i j}-k \mid}+\prod_{j=1}^{n}\left(\left(\varpi_{j}\right)^{2}\right)^{I\left(p_{i j}\right) r_{r_{j}}-k \mid} \leq 1 .
\end{aligned}
$$

It is known that $0 \leq \delta_{i}^{k} \leq 1,0 \leq \sigma_{i}^{k} \leq 1$, and $0 \leq\left(\delta_{i}^{k}\right)^{2}+\left(\sigma_{i}^{k}\right)^{2} \leq 1$. Hence, $d_{i}^{k}$ is a $\mathrm{PF}$ value. This completes the proof.

Definition 8. Let $d_{i}^{k}=\left(\delta_{i}^{k}, \sigma_{i}^{k}\right)$ be the comprehensive discordance indicator for an alternative $z_{i} \in Z$ to be assigned to a comprehensive rank $k$, where $k=1,2, \cdots, m$. The comprehensive discordance index $D\left(d_{i}^{k}\right)$ corresponding to each $d_{i}^{k}$ is defined as follows:

$$
D\left(d_{i}^{k}\right)=\frac{1+\left(\delta_{i}^{k}\right)^{2}-\left(\sigma_{i}^{k}\right)^{2}}{2} .
$$

Theorem 7. The comprehensive discordance index $D\left(d_{i}^{k}\right)$ of $d_{i}^{k}$ satisfies the following properties:

(T7.1) $0 \leq D\left(d_{i}^{k}\right) \leq 1$;

(T7.2) $D\left(d_{i}^{k}\right)=0$ if $d_{i}^{k}=(0,1)$;

(T7.3) $D\left(d_{i}^{k}\right)=1$ if $d_{i}^{k}=(1,0)$;

(T7.4) $D\left(d_{i}^{k}\right)=0.5$ if $d_{i}^{k}=(0,0)$;

(T7.5) $D\left(d_{i}^{k}\right)=\left(\delta_{i}^{k}\right)^{2}$ if $d_{i}^{k}=\left(\delta_{i}^{k}, \sqrt{1-\left(\delta_{i}^{k}\right)^{2}}\right)$.

Proof. (T7.1) According to $0 \leq \delta_{i}^{k} \leq 1,0 \leq \sigma_{i}^{k} \leq 1$, and $0 \leq\left(\delta_{i}^{k}\right)^{2}+\left(\sigma_{i}^{k}\right)^{2} \leq 1$, it can be obtained that $\left(\delta_{i}^{k}\right)^{2}-\left(\sigma_{i}^{k}\right)^{2} \leq\left(\delta_{i}^{k}\right)^{2} \leq 1$ and $\left(\delta_{i}^{k}\right)^{2}-\left(\sigma_{i}^{k}\right)^{2} \geq-\left(\sigma_{i}^{k}\right)^{2}$ $\geq-1$. Because $-1 \leq\left(\delta_{i}^{k}\right)^{2}-\left(\sigma_{i}^{k}\right)^{2} \leq 1$, one can conclude that $0 \leq D\left(d_{i}^{k}\right) \leq 1$. Thus, (T7.1) is valid.

(T7.2)-(T7.5) are straightforward, which completes the proof.

To arrive at a comprehensive ranking of alternatives, this paper constructs a novel assignment model to propose an assignment-based MCDA method. Let $\gamma_{i}^{k}$ denote a binary variable that is restricted to be either 0 or 1 . Let $\Gamma$ denote a permutation matrix whose element $\gamma_{i}^{k}=1$ if $z_{i}$ is assigned to the comprehensive rank $k$; otherwise, $\gamma_{i}^{k}=0$. Note that $\sum_{k=1}^{m} \gamma_{i}^{k}=1$ (i.e., $z_{i}$ should be assigned to only one rank in the comprehensive ranking) and $\sum_{i=1}^{m} \gamma_{i}^{k}=1$ (i.e., a given rank $k$ should only have one alternative assigned to it). $\Gamma$ is expressed as follows:

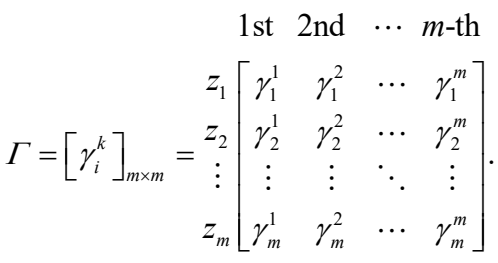

To show the usefulness of the permutation matrix $\Gamma$, consider the following simple example. Assume that there are three candidate alternatives $z_{1}, z_{2}$, and $z_{3}$. For convenience, let $\mathrm{Z}=\left(z_{1}, z_{2}, z_{3}\right)$ in this reference order. The ordering $\left(z_{3}, z_{1}, z_{2}\right)$ (i.e., the ranking $z_{3} \succ z_{1} \succ z_{2}$ ) could be obtained by multiplying $\mathrm{Z}$ by $\Gamma$, where

$$
\begin{array}{r}
\text { 1st } 2 \text { nd } 3 \text { rd } \\
\Gamma=\left[\gamma_{i}^{k}\right]_{3 \times 3}=z_{1}\left[\begin{array}{ccc}
0 & 1 & 0 \\
z_{2} & 0 & 0 \\
z_{3} \\
1 & 0 & 0
\end{array}\right] .
\end{array}
$$

This gives that:

$$
\mathrm{Z} \cdot \Gamma=\left(z_{1}, z_{2}, z_{3}\right) \cdot\left[\begin{array}{lll}
0 & 1 & 0 \\
0 & 0 & 1 \\
1 & 0 & 0
\end{array}\right]=\left(z_{3}, z_{1}, z_{2}\right) .
$$

Therefore, by varying the position of the zeroes and ones in the permutation matrix $\Gamma$, all of the possible rank orders can be generated correspondingly.

As far as the objective function in the developed assignment model is concerned, one can see that it combines the criterion-wise precedence rankings linearly by means of comparisons of the correlationbased precedence indices. Nevertheless, the overall compensation hypothesis among criteria is captured by the choice of the sum of comprehensive discordance indices which is to be minimized. More specifically, the decision maker would like to determine an optimal comprehensive ranking that effects a best compromise among all criterion-wise precedence rankings. Namely, the objective function should assign an alternative to that rank order which has the smallest comprehensive discordance index.

Reconsider the reference order $\mathrm{Z}=\left(z_{1}, z_{2}, z_{3}\right)$ as an example. Suppose that the following comprehensive discordance indices are obtained: $D\left(d_{1}^{1}\right)=0.92$, $D\left(d_{1}^{2}\right)=0.83, \quad D\left(d_{1}^{3}\right)=0.77, \quad D\left(d_{2}^{1}\right)=0.85, \quad D\left(d_{2}^{2}\right)=$ $0.76, D\left(d_{2}^{3}\right)=0.94, D\left(d_{3}^{1}\right)=0.75, D\left(d_{3}^{2}\right)=0.88$, and $D\left(d_{3}^{3}\right)=0.86$. If one arbitrarily chooses the ranking $z_{3} \succ z_{1} \succ z_{2}$, then the binary variables in $\Gamma$ are as follows: $\gamma_{1}^{2}=\gamma_{2}^{3}=\gamma_{3}^{1}=1$ and $\gamma_{1}^{1}=\gamma_{1}^{3}=\gamma_{2}^{1}=\gamma_{2}^{2}=\gamma_{3}^{2}=$ 
$\gamma_{3}^{3}=0$. Accordingly, the sum of the comprehensive discordance indices corresponding to the ordering $\left(z_{3}, z_{1}\right.$, $\left.z_{2}\right)$ is $2.52\left(=\sum_{i=1}^{3} \sum_{k=1}^{3}\left(\gamma_{i}^{k} \cdot D\left(d_{i}^{k}\right)\right)=D\left(d_{1}^{2}\right)+D\left(d_{2}^{3}\right)+\right.$ $\left.D\left(d_{3}^{1}\right)=0.83+0.94+0.75\right)$. Generally, the decision maker would think of examining all criterion-wise precedence rankings and choosing the one which yields the smallest value of $\sum_{i=1}^{3} \sum_{k=1}^{3}\left(\gamma_{i}^{k} \cdot D\left(d_{i}^{k}\right)\right)$. Because the ordering $\left(z_{3}, z_{2}, z_{1}\right)$ yields the smallest value 2.28 (= $\left.D\left(d_{1}^{3}\right)+D\left(d_{2}^{2}\right)+D\left(d_{3}^{1}\right)=0.77+0.76+0.75\right)$, the optimal comprehensive ranking in this example is $z_{3} \succ z_{2} \succ z_{1}$.

The smaller the degree of discordance indicated by $D\left(d_{i}^{k}\right)$ is, the greater the concordance will be from assigning $z_{i}$ to the $k$-th comprehensive rank. It is clear to see that the decision maker prefers that overall ranking where $\sum_{i=1}^{m} \sum_{k=1}^{m}\left(\gamma_{i}^{k} \cdot D\left(d_{i}^{k}\right)\right)$ is the smallest because it represents that ranking which effects the best compromise among all criterion-wise precedence rankings. Such an exhaustive search can be formulated using a simple linear programming model. Simply note that $\gamma_{i}^{k}$ is unknown to be determined by the model. Consequently, the constraints on the problem are the constraints for $\gamma_{i}^{k}$. Remember that $\sum_{k=1}^{m} \gamma_{i}^{k}=1$ and $\sum_{i=1}^{m} \gamma_{i}^{k}=1$. For solution purposes, the following assignment model can be established:

$$
\begin{aligned}
& \min \left\{\sum_{i=1}^{m} \sum_{k=1}^{m}\left(\gamma_{i}^{k} \cdot D\left(d_{i}^{k}\right)\right)\right\} \\
& \text { subject to } \sum_{k=1}^{m} \gamma_{i}^{k}=1, \quad i=1,2, \cdots, m, \\
& \sum_{i=1}^{m} \gamma_{i}^{k}=1, \quad k=1,2, \cdots, m, \\
& \gamma_{i}^{k}=0 \text { or } 1 \text { for all } i \text { and } k .
\end{aligned}
$$

Another way is to arbitrarily choose each of the $m$ ! possible rank orders to scrutinize which one yields the smallest value of $\sum_{i=1}^{m} \sum_{k=1}^{m}\left(\gamma_{i}^{k} \cdot D\left(d_{i}^{k}\right)\right)$. However, a linear programming procedure affects a solution easier and quicker. More precisely, solving the linear programming problem given by Eq. (23) yields the optimal permutation matrix $\hat{\Gamma}$ as would be obtained by complete enumeration but much more efficiently.

It can be verified that the $\hat{\Gamma}$ yields the smallest value of the total sum of the comprehensive discordance indices as required. Let the reference order $\mathrm{Z}=\left(z_{1}, z_{2}, \cdots, z_{m}\right)$ for notational convenience. Based on Eq. (24), the decision maker can find the optimal order by applying the permutation matrix $\hat{\Gamma}$ to Z. Namely, the optimal comprehensive ranks of the $m$ alternatives can be determined by multiplying $\mathrm{Z}$ by the optimal permutation matrix $\hat{\Gamma}$, as follows:

$$
\mathrm{Z} \cdot \hat{\Gamma}=\left(z_{1}, z_{2}, \cdots, z_{m}\right) \cdot\left[\begin{array}{cccc}
\hat{\gamma}_{1}^{1} & \hat{\gamma}_{1}^{2} & \cdots & \hat{\gamma}_{1}^{m} \\
\hat{\gamma}_{2}^{1} & \hat{\gamma}_{2}^{2} & \cdots & \hat{\gamma}_{2}^{m} \\
\vdots & \vdots & \ddots & \vdots \\
\hat{\gamma}_{m}^{1} & \hat{\gamma}_{m}^{2} & \cdots & \hat{\gamma}_{m}^{m}
\end{array}\right] .
$$

By using correlation-based precedence indices, the proposed assignment-based MCDA method can be summarized as follows:

- Step 1: Formulate an MCDA problem with the set of candidate alternatives $Z=\left\{z_{1}, z_{2}, \cdots, z_{m}\right\}$ and the set of criteria $C=\left\{c_{1}, c_{2}, \cdots, c_{n}\right\}$.

- Step 2: Establish the PF evaluative rating $p_{i j}$ of each alternative $z_{i} \in Z$ with respect to criterion $c_{j} \in C$ and the PF importance weight $w_{j}$ of each $c_{j}$. Form a PF decision matrix $\mathrm{p}=\left[p_{i j}\right]_{m \times n}$.

- Step 3: Apply Eq. (14) to calculate the correlationbased precedence index $I\left(p_{i j}\right)$ for each $p_{i j}$ in the PF decision matrix $\mathrm{p}$.

- Step 4: Form the rank evaluation matrix $r$ based on the descending order of the $I\left(p_{i j}\right)$ values with respect to each $c_{j}$. A mean rank should be assigned in case of the occurrence of ties.

- Step 5: Employ Eq. (20) to compute the comprehensive discordance indicator $d_{i}^{k}$ for $z_{i}$ to become the comprehensive rank $k$.

- Step 6: Use Eq. (21) to determine the comprehensive discordance index $D\left(d_{i}^{k}\right)$ of each $d_{i}^{k}$.

- Step 7: Construct a permutation matrix $\Gamma=$ $\left[\gamma_{i}^{k}\right]_{m \times m}$ and an assignment model using Eqs. (22) and (23), respectively.

- Step 8: Solve for the optimal permutation matrix $\hat{\Gamma}$. Obtain the optimal comprehensive ranks of the $m$ alternatives using Eq. (24).

\section{Practical Application}

As an application of the proposed methodology, this section investigates a financing decision problem on aggressive/conservative policies of working capital management for a medical institution to demonstrate the feasibility and effectiveness of the developed approach in practice.

Working capital whose main components are accounts payable, accounts receivable, and inventory concerns managing the day-to-day short-term operations of a firm. Working capital management has been 
playing a pivotal role for corporate finance due to its significant influence on firms' liquidity and profitability. Financing policy refers to the financing models implemented by firms to satisfy working capital requirements. The financing policy of working capital management can be generally determined as aggressive and conservative. Because of the particularity of the medical and health care system, financing decision on aggressive or conservative working capital policies becomes a highly complicated and ambiguous MCDA problem.

The medical institution investigated in this realworld case study is Chang Gung Memorial Hospital (CGMH). The main branch of CGMH is located in Linkou. Linkou CGMH is not only a multi-specialty hospital but also the largest medical center in Taiwan. Working capital management is important to Linkou CGMH because it can grant this medical center financial flexibility and reduce the dependence on external sources of finance. This case study comprises five plans related to working capital financing policy: aggressive dominant $\left(z_{1}\right)$ (i.e., $100 \%$ aggressive planoriented), aggressive-leaning $\left(z_{2}\right)$ (i.e., $75 \%$ aggressive and $25 \%$ conservative), balanced aggressive and conservative $\left(z_{3}\right)$ (i.e., $50 \%$ aggressive and 50\% conservative), conservative-leaning $\left(z_{4}\right)$ (i.e., 25\% aggressive and $75 \%$ conservative), and conservative dominant $\left(z_{5}\right)$ (i.e., 100\% conservative plan-oriented). To carefully evaluate the five types of alternative plans, managers consider the following six evaluation criteria: cash reserves $\left(c_{1}\right)$, maturity hedging $\left(c_{2}\right)$, interest rate fluctuation $\left(c_{3}\right)$, financial leverage $\left(c_{4}\right)$, return on assets $\left(c_{5}\right)$, and financing cost $\left(c_{6}\right)$.

The proposed assignment-based MCDA method using correlation-based precedence indices was employed to help Linkou CGMH to select the most appropriate financing policy of working capital management. In Step 1, this MCDA problem is defined by five financing policies and six criteria for evaluating the alternatives. The set of alternatives is denoted by $Z=\left\{z_{1}, z_{2}, \cdots, z_{5}\right\}$, and the set of criteria is denoted by $C=\left\{c_{1}, c_{2}, \cdots, c_{6}\right\}$.

In Step 2, based on managers' knowledge and expertise in Linkou CGMH, the PF decision matrix $\mathrm{p}$ $\left(=\left[p_{i j}\right]_{5 \times 6}\right)$ was constructed as follows:

$$
\begin{aligned}
& \begin{array}{lll}
c_{1} & c_{2} & c_{3}
\end{array} \\
& z_{1}\left[\begin{array}{llll}
(0.21,0.83) & (0.33,0.78) & (0.49,0.54)
\end{array}\right. \\
& z_{2}\left(\begin{array}{llll}
(0.36,0.74) & (0.55,0.48) & (0.61,0.42)
\end{array}\right. \\
& \mathrm{p}=z_{3}\left(\begin{array}{llll}
(0.63,0.52) & (0.77,0.25) \quad(0.88,0.21)
\end{array}\right. \\
& \begin{array}{l|lll}
z_{4} & (0.82,0.21) & (0.77,0.25) & (0.78,0.27)
\end{array} \\
& z_{5}\left[\begin{array}{lll}
(0.94,0.14) & (0.90,0.10) & (0.61,0.42)
\end{array}\right. \\
& \begin{array}{ccc}
c_{4} & c_{5} & c_{6}
\end{array} \\
& z_{1} \quad(0.29,0.70) \quad(0.91,0.13) \quad(0.49,0.40) \\
& z_{2} \quad(0.41,0.63) \quad(0.80,0.17) \quad(0.73,0.31) \\
& z_{3}(0.61,0.47) \quad(0.71,0.31) \quad(0.92,0.11) \\
& z_{4} \quad(0.74,0.31) \quad(0.32,0.79) \quad(0.84,0.18) \\
& \left.z_{5}(0.82,0.26) \quad(0.11,0.88) \quad(0.51,0.59)\right]
\end{aligned}
$$

The grades of criterion importance were expressed using a seven-point scale, and the obtained linguistic evaluation terms were as follows: low for $c_{1}$, fair for $c_{2}$, high for $c_{3}$, very low for $c_{4}$, very high for $c_{5}$, and extremely high for $c_{6}$. Applying Chen's seven-point

\begin{tabular}{|c|c|c|c|c|c|c|}
\hline$z_{i}$ & $c_{1}$ & $c_{2}$ & $c_{3}$ & $c_{4}$ & $c_{5}$ & $c_{6}$ \\
\hline & \multicolumn{6}{|c|}{ Computation results of $I\left(p_{i j}\right)$} \\
\hline$z_{1}$ & 0.0602 & 0.1518 & 0.4516 & 0.1465 & 0.9800 & 0.6001 \\
\hline$z_{2}$ & 0.1914 & 0.5676 & 0.6784 & 0.2975 & 0.9568 & 0.8472 \\
\hline$z_{3}$ & 0.5948 & 0.9046 & 0.9461 & 0.6275 & 0.8399 & 0.9859 \\
\hline$z 4$ & 0.9385 & 0.9046 & 0.8930 & 0.8507 & 0.1409 & 0.9561 \\
\hline \multirow[t]{2}{*}{$z 5$} & 0.9783 & 0.9878 & 0.6784 & 0.9086 & 0.0154 & 0.4277 \\
\hline & \multicolumn{6}{|c|}{ The original criterion-wise precedence ranks } \\
\hline$z_{1}$ & 5 & 5 & 5 & 5 & 1 & 4 \\
\hline$z_{2}$ & 4 & 4 & 3 & 4 & 2 & 3 \\
\hline$z_{3}$ & 3 & 2 & 1 & 3 & 3 & 1 \\
\hline$z_{4}$ & 2 & 2 & 2 & 2 & 4 & 2 \\
\hline$z_{5}$ & 1 & 1 & 3 & 1 & 5 & 5 \\
\hline
\end{tabular}
rating system $^{7}$, the $\mathrm{PF}$ importance weights of the six criteria were acquired as follows: $w_{1}=(0.35,0.65)$, $w_{2}=(0.55,0.45), \quad w_{3}=(0.65,0.35), \quad w_{4}=(0.25,0.75)$, $w_{5}=(0.75,0.25)$, and $w_{6}=(0.85,0.15)$.

In Step 3, the computed results of the correlationbased precedence index $I\left(p_{i j}\right)$ for each $p_{i j}$ in $\mathrm{p}$ are presented in the top part of Table 1 . Consider $I\left(p_{15}\right)$ as an example. By using Eq. (14), one can obtain:

$$
I\left(p_{15}\right)=\frac{\left(\mu_{15}\right)^{2}}{1-\left(\pi_{15}\right)^{2}}=\frac{0.91^{2}}{1-0.3937^{2}}=0.9800 \text {, }
$$

where $\pi_{15}=\sqrt{1-0.91^{2}-0.13^{2}}=0.3937$.

Table 1. Results of $I\left(p_{i j}\right)$ and the original precedence rank

In Step 4, as shown in the bottom part of Table 1, the original criterion-wise precedence ranks of all $z_{i}$ 
were obtained according to the descending order of the $I\left(p_{i j}\right)$ values. However, because $z_{3}$ and $z_{4}$ are tied at second place for $c_{2}$, a mean rank of $2.5(=(2+3) / 2)$ was given to both alternatives. Moreover, a mean rank of 3.5 $(=(3+4) / 2)$ was assigned to $z_{2}$ and $z_{5}$ because they are tied at third place for $c_{3}$. Thus, the rank evaluation matrix $\mathrm{r}\left(=\left[r_{i j}\right]_{5 \times 6}\right)$ was constructed as follows:

$$
\begin{aligned}
& \begin{array}{llllll}
c_{1} & c_{2} & c_{3} & c_{4} & c_{5} & c_{6}
\end{array} \\
& \mathrm{r}=z_{z_{3}} z_{z_{4}}\left[\begin{array}{cccccc}
z_{5} & 5 & 5 & 5 & 1 & 4 \\
4 & 4 & 3.5 & 4 & 2 & 3 \\
3 & 2.5 & 1 & 3 & 3 & 1 \\
2 & 2.5 & 2 & 2 & 4 & 2 \\
1 & 1 & 3.5 & 1 & 5 & 5
\end{array}\right] .
\end{aligned}
$$

Note that $\sum_{i=1}^{5} r_{i j}=15$ for all $j \in\{1,2, \cdots, 6\}$.

In Step 5, by using Eq. (20), the comprehensive discordance indicator $d_{i}^{k}$ was calculated, as shown in Table 2 .

Table 2. Results of $d_{i}^{k}$ and $D\left(d_{i}^{k}\right)$.

\begin{tabular}{ccccc}
\hline$z_{i}$ & $d_{i}^{k}$ & $D\left(d_{i}^{k}\right)$ & $d_{i}^{k}$ & $D\left(d_{i}^{k}\right)$ \\
\hline & 1st rank & 2nd rank \\
$z_{1}$ & $(0.9861,0.0023)$ & 0.9862 & $(0.9815,0.0036)$ & 0.9817 \\
$z_{2}$ & $(0.9952,0.0003)$ & 0.9952 & $(0.9395,0.0199)$ & 0.9411 \\
$z_{3}$ & $(0.9377,0.0138)$ & 0.9395 & $(0.9678,0.0080)$ & 0.9683 \\
$z_{4}$ & $(0.9669,0.0063)$ & 0.9674 & $(0.5718,0.4715)$ & 0.5523 \\
$z_{5}$ & $(0.9789,0.0060)$ & 0.9791 & $(0.9686,0.0065)$ & 0.9691 \\
& 3rd rank & & 4 th rank & \\
$z_{1}$ & $(0.9755,0.0056)$ & 0.9758 & $(0.9675,0.0087)$ & 0.9680 \\
$z_{2}$ & $(0.8405,0.0999)$ & 0.8483 & $(0.9708,0.0099)$ & 0.9712 \\
$z_{3}$ & $(0.9879,0.0023)$ & 0.9880 & $(0.9994,0.0000)$ & 0.9994 \\
$z_{4}$ & $(0.9414,0.0191)$ & 0.9429 & $(0.9930,0.0004)$ & 0.9930 \\
$z_{5}$ & $(0.9532,0.0070)$ & 0.9543 & $(0.9523,0.0037)$ & 0.9535 \\
& 5 th rank & & & \\
$z_{1}$ & $(0.9909,0.0014)$ & 0.9909 & & \\
$z_{2}$ & $(0.9976,0.0001)$ & 0.9976 & & \\
$z_{3}$ & $(1.0000,0.0000)$ & 1.0000 & & \\
$z_{4}$ & $(0.9993,0.0000)$ & 0.9993 & & \\
$z_{5}$ & $(0.9668,0.0010)$ & 0.9673 & & \\
\hline & & & & \\
& & & \\
\end{tabular}

Consider $z_{4}$ to be assigned the second comprehensive rank as an example. First, the product of $I\left(p_{4 j}\right)$ and $\left|r_{4 j}-2\right|$ was calculated for each $j=1,2, \cdots, 6$, and the following results were acquired: $I\left(p_{41}\right)\left|r_{41}-2\right|=0.9385 \cdot|2-2|=0 \quad, \quad I\left(p_{42}\right)\left|r_{42}-2\right|=$ $0.9046 \cdot|2.5-2|=0.4523, I\left(p_{43}\right)\left|r_{43}-2\right|=0.8930 \cdot \mid 2-$ $2\left|=0, I\left(p_{44}\right)\right| r_{44}-2|=0.8507 \cdot| 2-2\left|=0, I\left(p_{45}\right)\right| r_{45}$ $-2|=0.1409 \cdot| 4-2 \mid=0.2818$, and $I\left(p_{46}\right)\left|r_{46}-2\right|=$ $0.9561 \cdot|2-2|=0$. Then, one obtains:

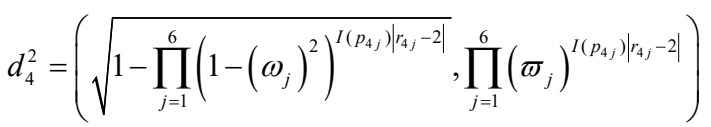

$$
\begin{aligned}
& =\left(\left[1-\left(1-0.35^{2}\right)^{0}\left(1-0.55^{2}\right)^{0.4523}\left(1-0.65^{2}\right)^{0}\right.\right. \\
& \left.\cdot\left(1-0.25^{2}\right)^{0}\left(1-0.75^{2}\right)^{0.2818}\left(1-0.85^{2}\right)^{0}\right]^{0.5} \text {, } \\
& \left.0.65^{0} \cdot 0.45^{0.4523} \cdot 0.35^{0} \cdot 0.75^{0} \cdot 0.25^{0.2818} \cdot 0.15^{0}\right) \\
& =(0.5718,0.4715) \text {. }
\end{aligned}
$$

In Step 6, by using Eq. (21), the comprehensive discordance index $D\left(d_{i}^{k}\right)$ of each $d_{i}^{k}$ was obtained, as revealed in Table 2. For example, $D\left(d_{4}^{2}\right)=\left(1+0.5718^{2}\right.$ $\left.-0.4715^{2}\right) / 2=0.5523$.

In Step 7, a permutation matrix $\Gamma$ whose entry $\gamma_{i}^{k} \in\{0,1\}$ for each $i, k \in\{1,2, \cdots, 5\}$ was established as follows:

$$
\begin{aligned}
& \text { 1st 2nd 3rd 4th 5th } \\
& \Gamma=z_{3}\left[\begin{array}{lllll}
z_{1} \\
z_{2} \\
z_{4} \\
z_{5}
\end{array}\left[\begin{array}{lllll}
\gamma_{1}^{1} & \gamma_{1}^{2} & \gamma_{1}^{3} & \gamma_{1}^{4} & \gamma_{1}^{5} \\
\gamma_{2}^{1} & \gamma_{2}^{2} & \gamma_{2}^{3} & \gamma_{2}^{4} & \gamma_{2}^{5} \\
\gamma_{3}^{1} & \gamma_{3}^{2} & \gamma_{3}^{3} & \gamma_{3}^{4} & \gamma_{3}^{5} \\
\gamma_{4}^{1} & \gamma_{4}^{2} & \gamma_{4}^{3} & \gamma_{4}^{4} & \gamma_{4}^{5} \\
\gamma_{5}^{1} & \gamma_{5}^{2} & \gamma_{5}^{3} & \gamma_{5}^{4} & \gamma_{5}^{5}
\end{array}\right] .\right.
\end{aligned}
$$

Moreover, by using Eq. (23), the following assignment model was constructed:

$$
\begin{aligned}
& \min \left\{\begin{array}{l}
0.9862 \cdot \gamma_{1}^{1}+0.9817 \cdot \gamma_{1}^{2}+0.9758 \cdot \gamma_{1}^{3} \\
+0.9680 \cdot \gamma_{1}^{4}+0.9909 \cdot \gamma_{1}^{5}+0.9952 \cdot \gamma_{2}^{1} \\
+0.9411 \cdot \gamma_{2}^{2}+0.8483 \cdot \gamma_{2}^{3}+0.9712 \cdot \gamma_{2}^{4} \\
+0.9976 \cdot \gamma_{2}^{5}+0.9395 \cdot \gamma_{3}^{1}+0.9683 \cdot \gamma_{3}^{2} \\
+0.9880 \cdot \gamma_{3}^{3}+0.9994 \cdot \gamma_{3}^{4}+1.0000 \cdot \gamma_{3}^{5} \\
+0.9674 \cdot \gamma_{4}^{1}+0.5523 \cdot \gamma_{4}^{2}+0.9429 \cdot \gamma_{4}^{3} \\
+0.9930 \cdot \gamma_{4}^{4}+0.9993 \cdot \gamma_{4}^{5}+0.9791 \cdot \gamma_{5}^{1} \\
+0.9691 \cdot \gamma_{5}^{2}+0.9543 \cdot \gamma_{5}^{3}+0.9535 \cdot \gamma_{5}^{4} \\
+0.9673 \cdot \gamma_{5}^{5}
\end{array}\right\} \\
& \text { subject to } \sum_{k=1}^{5} \gamma_{i}^{k}=1, \quad i=1,2, \cdots, 5 \text {, } \\
& \sum_{i=1}^{5} \gamma_{i}^{k}=1, k=1,2, \cdots, 5, \\
& \gamma_{i}^{k}=0 \text { or } 1 \text { for all } i \text { and } k .
\end{aligned}
$$


In Step 8, the optimal objective value 4.2754 was acquired by solving the above model; moreover, $\hat{\gamma}_{1}^{4}=\hat{\gamma}_{2}^{3}=\hat{\gamma}_{3}^{1}=\hat{\gamma}_{4}^{2}=\hat{\gamma}_{5}^{5}=1$, and the other $\hat{\gamma}_{i}^{k}=0$. By using (24), the optimal comprehensive ranks of the five financing policies were determined by multiplying $Z$ by $\hat{\Gamma}$, i.e.:

$$
\begin{aligned}
\mathrm{Z} \cdot \hat{\Gamma} & =\left(z_{1}, z_{2}, z_{3}, z_{4}, z_{5}\right) \cdot\left[\begin{array}{ccccc}
0 & 0 & 0 & 1 & 0 \\
0 & 0 & 1 & 0 & 0 \\
1 & 0 & 0 & 0 & 0 \\
0 & 1 & 0 & 0 & 0 \\
0 & 0 & 0 & 0 & 1
\end{array}\right] \\
& =\left(z_{3}, z_{4}, z_{2}, z_{1}, z_{5}\right) .
\end{aligned}
$$

From the above, it is clear that the optimal comprehensive ranking of the five financing policies is $z_{3} \succ z_{4} \succ z_{2} \succ z_{1} \succ z_{5}$ that has the least disagreement with all of the criterion-wise precedence rankings of the alternatives. Moreover, the balanced aggressive and conservative policy $\left(z_{3}\right)$ is the best choice for Linkou CGMH. Having illustrated the solution process, the feasibility and effectiveness of the proposed methodology have been validated through the practical application of a financing decision on working capital policies.

\section{Comparative discussion}

This section attempts to employ the assignment-based MCDA method using correlation-based precedence indices to investigate the application effects within the intuitionistic fuzzy environment. More specifically, the sensitivities of applying the develop approach over implementations of intuitionistic fuzzy information are further explored to examine the applicability and effectiveness in the context of intuitionistic fuzziness.

Consider the same financing decision problem. Inspired by the transformation procedure of interval type data introduced by $\mathrm{Chen}^{5}$, this paper converts the $\mathrm{PF}$ evaluative rating $p_{i j}$ and the PF importance weight $w_{j}$ into intuitionistic fuzzy values $\bar{p}_{i j}$ and $\bar{w}_{j}$, respectively, using the following manner:

$$
\begin{aligned}
\bar{p}_{i j} & =\left(\bar{\mu}_{i j}, \bar{v}_{i j}\right) \\
& =\left(\frac{\mu_{i j}}{\mu_{i j}+v_{i j}+\pi_{i j}}, \frac{v_{i j}}{\mu_{i j}+v_{i j}+\pi_{i j}}\right),
\end{aligned}
$$

$$
\bar{w}_{j}=\left(\bar{\omega}_{j}, \bar{\varpi}_{j}\right)=\left(\frac{\omega_{j}}{\omega_{j}+\varpi_{j}+\tau_{j}}, \frac{\varpi_{j}}{\omega_{j}+\varpi_{j}+\tau_{j}}\right),
$$

where $\bar{\pi}_{i j}=1-\bar{\mu}_{i j}-\bar{v}_{i j}$ and $\bar{\tau}_{j}=1-\bar{\omega}_{j}-\bar{\varpi}_{j}$

To adapt to the intuitionistic fuzzy context, some formulas in the proposed method have to be modified using intuitionistic fuzzy operations. First, the information energy of $\bar{p}_{i j}$ is computed as follows:

$$
T\left(\bar{p}_{i j}\right)=\left(\bar{\mu}_{i j}\right)^{2}+\left(\bar{v}_{i j}\right)^{2}+\left(\bar{\pi}_{i j}\right)^{2} .
$$

The correlation between $\bar{p}_{i_{1} j}$ and $\bar{p}_{i_{2} j}$ is given by:

$$
R\left(\bar{p}_{i_{1} j}, \bar{p}_{i_{2} j}\right)=\bar{\mu}_{i_{1} j} \cdot \bar{\mu}_{i_{2} j}+\bar{v}_{i_{1} j} \cdot \bar{v}_{i_{2} j}+\bar{\pi}_{i_{1} j} \cdot \bar{\pi}_{i_{2} j} .
$$

The correlation-based precedence index of $\bar{p}_{i j}$ is determined as follows:

$$
I\left(\bar{p}_{i j}\right)=\frac{\bar{\mu}_{i j}}{1-\bar{\pi}_{i j}} .
$$

Next, the comprehensive discordance indicator $\bar{d}_{i}^{k}$ in the intuitionistic fuzzy context is computed as follows:

$$
\bar{d}_{i}^{k}=\left(\sqrt{1-\prod_{j=1}^{n}\left(1-\bar{\omega}_{j}\right)^{I\left(\bar{p}_{i j}\right)\left|r_{i j}-k\right|}}, \prod_{j=1}^{n}\left(\bar{\varpi}_{j}\right)^{I\left(\bar{p}_{i j}\right)\left|r_{i j}-k\right|}\right)
$$

The comprehensive discordance index $D\left(\bar{d}_{i}^{k}\right)$ corresponding to each $\bar{d}_{i}^{k}$ is defined as follows:

$$
D\left(\bar{d}_{i}^{k}\right)=\frac{1+\bar{\delta}_{i}^{k}-\bar{\sigma}_{i}^{k}}{2} .
$$

Table 3 reveals the obtained results of the correlation-based precedence index $I\left(\bar{p}_{i j}\right)$, as shown in the top part. The adjusted criterion-wise precedence ranks are listed in the bottom part. Furthermore, the computed results of $\bar{d}_{i}^{k}$ and $D\left(\bar{d}_{i}^{k}\right)$ in the intuitionistic fuzzy context are depicted in Table 4.

Table 3. Results of $I\left(\bar{p}_{i j}\right)$ and the original precedence rank.

\begin{tabular}{ccccccc}
\hline$z_{i}$ & $c_{1}$ & $c_{2}$ & $c_{3}$ & $c_{4}$ & $c_{5}$ & $c_{6}$ \\
\hline \multicolumn{7}{c}{ Computation results of $I\left(\bar{p}_{i j}\right)$} \\
$z_{1}$ & 0.0602 & 0.1518 & 0.4516 & 0.1465 & 0.9800 & 0.6001 \\
$z_{2}$ & 0.1914 & 0.5676 & 0.6784 & 0.2975 & 0.9568 & 0.8472 \\
$z_{3}$ & 0.5948 & 0.9046 & 0.9461 & 0.6275 & 0.8399 & 0.9859 \\
$z_{4}$ & 0.9385 & 0.9046 & 0.8930 & 0.8507 & 0.1409 & 0.9561 \\
$z_{5}$ & 0.9783 & 0.9878 & 0.6784 & 0.9086 & 0.0154 & 0.4277 \\
\multicolumn{7}{c}{ The adjusted criterion-wise precedence ranks } \\
$z_{1}$ & 5 & 5 & 5 & 5 & 1 & 4 \\
$z_{2}$ & 4 & 4 & 3.5 & 4 & 2 & 3 \\
$z_{3}$ & 3 & 2.5 & 1 & 3 & 3 & 1 \\
$z_{4}$ & 2 & 2.5 & 2 & 2 & 4 & 2 \\
$z_{5}$ & 1 & 1 & 3.5 & 1 & 5 & 5 \\
\hline \multicolumn{7}{c}{}
\end{tabular}


Table 4. Results of $\bar{d}_{i}^{k}$ and $D\left(\bar{d}_{i}^{k}\right)$.

\begin{tabular}{ccccc}
\hline$z_{i}$ & $\bar{d}_{i}^{k}$ & $D\left(\bar{d}_{i}^{k}\right)$ & $\bar{d}_{i}^{k}$ & $D\left(\bar{d}_{i}^{k}\right)$ \\
\hline & 1st rank & & 2nd rank \\
$z_{1}$ & $(0.9576,0.0000)$ & 0.9788 & $(0.9394,0.0001)$ & 0.9696 \\
$z_{2}$ & $(0.9688,0.0000)$ & 0.9844 & $(0.8215,0.0035)$ & 0.9090 \\
$z_{3}$ & $(0.8280,0.0025)$ & 0.9127 & $(0.8572,0.0023)$ & 0.9274 \\
$z_{4}$ & $(0.9035,0.0006)$ & 0.9515 & $(0.3983,0.2066)$ & 0.5959 \\
$z_{5}$ & $(0.9218,0.0006)$ & 0.9606 & $(0.9167,0.0004)$ & 0.9582 \\
& 3rd rank & & 4th rank & \\
$z_{1}$ & $(0.9134,0.0005)$ & 0.9564 & $(0.8762,0.0016)$ & 0.9373 \\
$z_{2}$ & $(0.6369,0.0361)$ & 0.8004 & $(0.8283,0.0058)$ & 0.9113 \\
$z_{3}$ & $(0.9117,0.0008)$ & 0.9554 & $(0.9891,0.0000)$ & 0.9946 \\
$z_{4}$ & $(0.8142,0.0045)$ & 0.9048 & $(0.9573,0.0000)$ & 0.9786 \\
$z_{5}$ & $(0.9113,0.0003)$ & 0.9555 & $(0.9293,0.0001)$ & 0.9646 \\
& 5 th rank & & & \\
$z_{1}$ & $(0.9292,0.0004)$ & 0.9644 & & \\
$z_{2}$ & $(0.9699,0.0000)$ & 0.9850 & & \\
$z_{3}$ & $(0.9987,0.0000)$ & 0.9993 & & \\
$z_{4}$ & $(0.9931,0.0000)$ & 0.9966 & & \\
$z_{5}$ & $(0.9579,0.0000)$ & 0.9790 & & \\
\hline
\end{tabular}

By solving the assignment model based on Eq. (23), the same optimal comprehensive ranking $z_{3} \succ z_{4} \succ z_{2}$ $\succ z_{1} \succ z_{5}$ is obtained. According to the implementation results of the sensitivities with intuitionistic fuzzy information, this paper has demonstrated that the developed approach can yield solid and conscious results to support decision makers. In addition to the PF environment, the proposed methodology is also capable of addressing MCDA problems involving intuitionistic fuzzy information and producing reasonable and reliable outcomes for decision support.

\section{Conclusions}

This paper has developed a novel assignment-based MCDA method using correlation-based precedence indices for the purpose of managing vague or imprecise information and conducting multiple criteria evaluation and selection under complex uncertainty based on PF sets. The theory of Pythagorean fuzziness can accommodate much higher degrees of uncertainty compared to other nonstandard fuzzy models. Therefore, PF sets are more powerful at solving complicated and changeable real-world problems. Accordingly, the PF theory becomes popular and useful for solving various MCDA issues over the past few years.

However, it has been observed that the mathematical formulation and operations required in the PF relevant approaches have certain limitations. Specifically, the main drawback of the existing PF approaches is the manipulation and computation of complicated PF data. In general, using considerate and rigorous models and techniques to process PF information is a troublesome and difficult task for most decision makers. To overcome the difficulty during mathematical manipulation, this paper has proposed easy-to-use measures of PF correlation coefficients and further established a simple assignment-based model for managing an MCDA problem with PF information.

Inspired by the idea of the correlation coefficient for PF sets, this paper has presented some useful concepts of the information energy of a PF value, the correlation between two PF values, and the correlation coefficient for PF values in order to identify the correlation-based precedence index. Meanwhile, relevant desirable properties possessed by these concepts have been discussed and investigated as well.

In addition to the correlation-based precedence index, this paper has proposed some interesting and valuable measures within the PF environment, consisting of the discordance indicator, the weighted discordance indicator, the comprehensive discordance indicator, and the comprehensive discordance index. Based on these indicators and the comprehensive discordance index, a simple and effective assignment model has been established to determine the optimal comprehensive ranking among candidate alternatives.

For the sake of illustrative applications, a real-world problem concerning a financing decision on working capital policies for Linkou CGMH has been explored. The application results have examined the practicality and effectiveness of the developed methods and techniques that are capable of managing more-complex uncertainty in practice.

\section{Acknowledgements}

The authors are grateful for grant funding support from the Taiwan Ministry of Science and Technology (MOST 105-2410-H-182-007-MY3) and Chang Gung Memorial Hospital (BMRP 574 and CMRPD2F0202) during the completion of this study.

\section{References}

1. E. Afful-Dadzie, Z. K. Oplatková and L. A. Beltran Prieto, Comparative state-of-the-art survey of classical fuzzy set and intuitionistic fuzzy sets in multi-criteria decision making, International Journal of Fuzzy Systems 19(3) (2017) 726-738. https://doi.org/10.1007/s40815016-0204-y 
2. K. T. Atanassov, Intuitionistic fuzzy sets, Fuzzy Sets and Systems 20(1) (1986) 87-96. https://doi.org/10.1016/ S0165-0114(86)80034-3

3. T. Bao, X. Xie, P. Long and Z. Wei, MADM method based on prospect theory and evidential reasoning approach with unknown attribute weights under intuitionistic fuzzy environment, Expert Systems with Applications 88 (2018) 305-317. https://doi.org/10.1016/ j.eswa.2017.07.012

4. G. Büyüközkan and S. Güleryüz, Multi criteria group decision making approach for smart phone selection using intuitionistic fuzzy TOPSIS, International Journal of Computational Intelligence Systems 9(4) (2016) 709725. https://doi.org/10.1080/18756891.2016.1204119

5. T.-Y. Chen, A novel VIKOR method with an application to multiple criteria decision analysis for hospital-based post-acute care within a highly complex uncertain environment, Neural Computing \& Applications (2018). (in press) DOI: 10.1007/s00521-017-3326-8

6. T.-Y. Chen, An interval-valued Pythagorean fuzzy outranking method with a closeness-based assignment model for multiple criteria decision making, International Journal of Intelligent Systems 33(1) (2018) 126-168. DOI: 10.1002/int.21943

7. T.-Y. Chen, Remoteness index-based Pythagorean fuzzy VIKOR methods with a generalized distance measure for multiple criteria decision analysis, Information Fusion 41 (2018) 129-150. https://doi.org/10.1016/j.inffus.2017. 09.003

8. Y. Du, F. Hou, W. Zafar, Q. Yu and Y. Zhai, A novel method for multiattribute decision making with interval-valued Pythagorean fuzzy linguistic information, International Journal of Intelligent Systems 32(10) (2017) 1085-1112. DOI: 10.1002/int.21881

9. H. Garg, A novel correlation coefficient between Pythagorean fuzzy sets and its applications to decisionmaking processes, International Journal of Intelligent Systems 31(12) (2016) 1234-1252. DOI: 10.1002/int. 21827

10. H. Garg, Generalized Pythagorean fuzzy geometric aggregation operators using Einstein t-norm and tconorm for multicriteria decision-making process, International Journal of Intelligent Systems 32(6) (2017) 597-630. DOI: 10.1002/int.21860

11. C. Kahraman, B. Öztayşi and S. Çevik Onar, A comprehensive literature review of 50 years of fuzzy set theory, International Journal of Computational Intelligence Systems 9 (2016) 3-24. https://doi.org/ $10.1080 / 18756891.2016 .1180817$

12. D. Liang and $\mathrm{Z}$. Xu, The new extension of TOPSIS method for multiple criteria decision making with hesitant Pythagorean fuzzy sets, Applied Soft Computing 60 (2017) 167-179. https://doi.org/10.1016/j.asoc.2017. 06.034

13. V. Mohagheghi, S. M. Mousavi and B. Vahdani, Enhancing decision-making flexibility by introducing a new last aggregation evaluating approach based on multi- criteria group decision making and Pythagorean fuzzy sets, Applied Soft Computing 61 (2017) 527-535. https://doi.org/10.1016/j.asoc.2017.08.003

14. X. Peng and Y. Yang, Some results for Pythagorean fuzzy sets, International Journal of Intelligent Systems 30(11) (2015) 1133-1160. DOI: 10.1002/int.21738

15. X. Peng, H. Yuan and Y. Yang, Pythagorean fuzzy information measures and their applications, International Journal of Intelligent Systems 32(10) (2017) 991-1029. DOI: 10.1002/int.21880

16. K. Rahman, M. A. Khan, M. Ullah and A. Fahmi, Multiple attribute group decision making for plant location selection with Pythagorean fuzzy weighted geometric aggregation operator, The Nucleus 54(1) (2017) 66-74.

17. P. Ren, Z. Xu and X. Gou, Pythagorean fuzzy TODIM approach to multi-criteria decision making, Applied Soft Computing 42 (2016) 246-259. https://doi.org/10.1016/ j.asoc.2015.12.020

18. H. Wang and Z. Xu, Multi-groups decision making using intuitionistic-valued hesitant fuzzy information, International Journal of Computational Intelligence Systems 9(3) (2016) 468-482. https://doi.org/10.1080/ 18756891.2016.1175812

19. G. Wei, Pythagorean fuzzy interaction aggregation operators and their application to multiple attribute decision making, Journal of Intelligent and Fuzzy Systems 33(4) (2017) 2119-2132. DOI: 10.3233/JIFS162030

20. G. Wei and M. Lu, Pythagorean fuzzy power aggregation operators in multiple attribute decision making, International Journal of Intelligent Systems 33(1) (2018) 169-186. DOI: 10.1002/int.21946

21. Q. Xu, K. Yu, S. Zeng and J. Liu, Pythagorean fuzzy induced generalized OWA operator and its application to multi-attribute group decision making, International Journal of Innovative Computing, Information and Control 13(5) (2017) 1527-1536.

22. W. Xue, Z. Xu, X. Zhang and X. Tian, Pythagorean fuzzy LINMAP method based on the entropy theory for railway project investment decision making, International Journal of Intelligent Systems 33(1) (2018) 93-125. DOI: 10.1002/int.21941

23. R. R. Yager, Pythagorean membership grades in multicriteria decision making, IEEE Transactions on Fuzzy Systems 22(4) (2014) 958-965. DOI: 10.1109/ TFUZZ.2013.2278989

24. R. R. Yager and A. M. Abbasov, Pythagorean membership grades, complex numbers, and decision making, International Journal of Intelligent Systems 28(5) (2013) 436-452. DOI: 10.1002/int.21584

25. X. Zhang, Multicriteria Pythagorean fuzzy decision analysis: a hierarchical QUALIFLEX approach with the closeness index-based ranking methods, Information Sciences 330 (2016) 104-124. https://doi.org/10.1016/ j.ins.2015.10.012 